\title{
Electronic Energy Spectra of Square and Cubic Fibonacci Quasicrystals
}

\author{
SHAHAR EVEN-DAR MANDEL and RON LIFSHITZ \\ Raymond and Beverly Sackler School of Physics and Astronomy \\ Tel Aviv University, Tel Aviv 69978, Israel
}

December 18, 2007

\begin{abstract}
Understanding the electronic properties of quasicrystals, in particular the dependence of these properties on dimension, is among the interesting open problems in the field of quasicrystals. We investigate an off-diagonal tight-binding hamiltonian on the separable square and cubic Fibonacci quasicrystals. We use the well-studied singular-continuous energy spectrum of the 1-dimensional Fibonacci quasicrystal to obtain exact results regarding the transitions between different spectral behaviors of the square and cubic quasicrystals. We use analytical results for the addition of $1 d$ spectra to obtain bounds on the range in which the higher-dimensional spectra contain an absolutely continuous component. We also perform a direct numerical study of the spectra, obtaining good results for the square Fibonacci quasicrystal, and rough estimates for the cubic Fibonacci quasicrystal.
\end{abstract}

\section{Background and Motivation}

As we celebrate the Silver Jubilee of the 1982 discovery of quasicrystals [1], and highlight the achievements of the past two and a half decades of research on quasicrystals, we are reminded that there still remains a disturbing gap in our understanding of their electronic properties. Among the open questions is a lack of understanding of the dependence of electronic properties - such as the nature of electronic wave functions, their energy spectra, and the nature of electronic transport - on the dimension of the quasicrystal. In an attempt to bridge some of this gap, we $[2,3]$ have been studying the spectrum and electronic wave functions of an off-diagonal tight-binding hamiltonian on the separable $n$-dimensional Fibonacci quasicrystals 1 [4]. The advantage of using such separable models, despite the fact that they do not occur in nature, is the ability to obtain exact results in one, two, and three dimensions, and compare them directly to each other. Here we focus on the energy spectra of the 2-dimensional $(2 d)$ and 3-dimensional (3d) Fibonacci quasicrystals to obtain a quantitative understanding of the nature of the transitions between different spectral behaviors in these crystals, as their dimension increases from 1 up to 3 . In particular, we consider the transitions between different regimes in the spectrum, taking into account the existence of a regime in which the spectrum contains both singular continuous

\footnotetext{
${ }^{1}$ The reader is referred to Refs. [5] and [6] for precise definitions of the terms 'crystal' and 'quasicrystal'.
} 
and absolutely continuous components. These different behaviors of the higher-dimensional spectra are expected to reflect on the physical extent of the electronic wave functions, as well as on the dynamics of electronic wave packets, and are therefore of great importance in unraveling the electronic properties of quasicrystals in general.

Recall [2] that the off-diagonal tight-binding model assumes equal on-site energies (taken to be zero), and hopping that is restricted along tile edges, with amplitude 1 for long $(L)$ edges and $T$ for short $(S)$ edges, where we take $T \geq 1$. The Schrödinger equation for the square Fibonacci quasicrystal in $2 d$ (with obvious extensions to higher dimensions) is then given by

$$
T_{n+1} \Psi(n+1, m)+T_{n} \Psi(n-1, m)+T_{m+1} \Psi(n, m+1)+T_{m} \Psi(n, m-1)=E \Psi(n, m),
$$

where $\Psi(n, m)$ is the value of a $2 d$ eigenfunction on a vertex labeled by the two integers $n$ and $m$, and $E$ is the corresponding eigenvalue. The hopping amplitudes $T_{j}$ are equal to 1 or $T$ according to the Fibonacci sequence $\left\{T_{j}\right\}=\{1, T, 1,1, T, 1, T, 1,1, T, 1,1, T, 1, T, 1,1, T, 1, T, \ldots\}$. By prohibiting diagonal hopping, the resulting high-dimensional eigenvalue problem is ensured to be separable. This allows one to use the known solutions for the $1 d$ problem [7-14] in order to construct the solutions in two and higher dimensions (as was done for similar models in the past [15-21]). Two-dimensional eigenfunctions can therefore be expressed as Cartesian products of the $1 d$ eigenfunctions [3], and the corresponding $2 d$ eigenvalues are given by pairwise sums of the $1 d$ eigenvalues.

The $1 d$ spectrum for the $N^{\text {th }}$ order Fibonacci approximant is composed of $F_{N}$ bands, where $F_{N}=$ $F_{N-1}+F_{N-2}$ is the $N^{t h}$ Fibonacci number, starting with $F_{0}=F_{1}=1$. The edges of each such band correspond to either periodic or antiperiodic boundary conditions. Hence, by direct diagonalization of the two corresponding hamiltonians for a single approximant we obtain the edges of the energy intervals in the spectrum. The $2 d$ and $3 d$ spectra are then calculated as the Minkowski sums of two or three $1 d$ spectra, where the Minkowski sum of two sets $A$ and $B$ is the result of adding every element of $A$ to every element of $B$, i.e. the set

$$
A+B=\{x+y \mid x \in A, y \in B\} .
$$

Although the spectrum of the $1 d$ Fibonacci model, for any choice of $T \neq 1$, is a totally disconnected set with zero bandwidth and an infinite number of bands, the higher-dimensional spectra exhibit different behavior for different values of the relative hopping parameter $T$, including spectra that contain continuous intervals and have a finite measure [2]. A similar situation arises in the case of the well-known ternary Cantor set [22], which is constructed iteratively by starting with the closed interval $[0,1]$, and at each iteration removing the open middle thirds of all remaining closed intervals from the previous iteration. The first few approximants that are obtained in this way are $C_{0}=[0,1]$, $C_{1}=[0,1 / 3] \cup[2 / 3,1]$, and $C_{2}=[0,1 / 9] \cup[2 / 9,1 / 3] \cup[2 / 3,7 / 9] \cup[8 / 9,1]$, so that after $N$ such iterations one is left with an approximant set $C_{N}$ consisting of $2^{N}$ closed intervals, each of which has a measure $1 / 3^{N}$, and therefore the total measure of the set is $(2 / 3)^{N}$. The ternary Cantor set itself $C_{\infty}$, defined as the limit $N \rightarrow \infty$ of this sequence of sets, contains uncountably-many points yet no interval, it is totally disconnected, and its total measure is zero. By simple inspection, one finds that for any finite order Cantor approximant $C_{N}$, the Minkowski sum $C_{N}+C_{N}$ is the entire interval $[0,2]$. One can 
show that this also holds in the limit $N \rightarrow \infty$, namely that $C_{\infty}+C_{\infty}=[0,2]$. Thus, even though $C_{\infty}$ contains no interval, its sum with itself covers the whole interval from 0 to 2.

For a given dimension $n$, we identify a sequence of values $1<T_{1}^{(n d)} \leq T_{2}^{(n d)} \leq T_{3}^{(n d)} \leq T_{4}^{(n d)}$ corresponding to the following transitions in the spectrum:

$T_{1}^{(n d)}$ : The value of $T$ below which all bands in the $n$-dimensional spectrum are of positive, finite measure. For $T>T_{1}^{(n d)}$ there is at least a finite number of zero measure bands in the spectrum.

$T_{2}^{(n d)}$ : The value of $T$ above which the number of bands in the $n$-dimensional spectrum is infinite. An infinite number of bands in a spectrum of finite bandwidth necessarily implies that infinitely many bands are of zero measure, thus $T_{2}^{(n d)} \geq T_{1}^{(n d)}$.

$T_{3}^{(n d)}$ : The value of $T$ above which all bands in the spectrum are of zero measure 2

$T_{4}^{(n d)}$ : The value of $T$ above which the total bandwidth of the spectrum is zero.

We use two different approaches to study the behavior of the spectrum. In Sec. 2 we use analytical results derived for the addition of generalized Cantor sets to obtain an upper bound on the transition between absolutely continuous and singular continuous spectra. In Sec. 3 we use direct numerical calculation of the $2 d$ and $3 d$ spectra of Fibonacci approximants of finite order to extrapolate for the behavior in the quasiperiodic limit. In an earlier paper [3] we studied only two of the transitions, $T_{2}^{(n d)}$ and $T_{4}^{(n d)}$. To find $T_{4}^{(n d)}$ we used a naive method based on the results of Ashraff et al. [21] for the diagonal tight-binding hamiltonian. The current results include a correction to our previous calculation. In Sec. 4 we summarize the results, and discuss their expected relation to the nature of eigenfunctions and to quantum dynamics, indicating directions for future work.

\section{Analytical bounds for the appearance of continuous intervals in the spectrum}

\subsection{Addition of generalized Cantor sets - Known results}

A generalized Cantor set is obtained just like the ternary Cantor set except that the open intervals removed at each iteration are not necessarily the middle thirds of the remaining closed intervals. For each interval removed from the set, one defines a left (right) ratio of dissection as the ratio between the length of the left (right) remaining interval and the length of the original one. Sets for which the left and right ratios are the same are called central Cantor sets. In general, the ratios of dissection may vary between the left and right resulting intervals, between different iterations of the process, and between different intervals at the same step. The ternary Cantor set is a central Cantor set with a constant ratio of dissection of $1 / 3$.

We are interested in conditions for the appearance of intervals in the Minkowski sum of $n$ generalized Cantor sets. For central Cantor sets with a constant ratio of dissection $a$, one can show that the

\footnotetext{
${ }^{2}$ Note that the absence of intervals in the spectrum above $T_{3}^{(n d)}$ does not necessarily correspond to zero total bandwidth. It is in fact possible to use the Cantor set generation process to obtain a totally disconnected set with a finite measure. For example, if at the $N^{\text {th }}$ iteration of the generation process the middle $1 / 3^{N}$ part is removed from each of the remaining intervals, one ends with a totally disconnected set whose measure is $\lim \prod_{k=1}^{\infty}\left(1-1 / 3^{k}\right) \simeq 0.5601$.
} 
condition for the sum to be an interval is

$$
n \frac{a}{1-a} \geq 1 \quad \text { or } \quad a \geq \frac{1}{n+1} .
$$

Thus, the ternary Cantor set exactly has the critical value of $a=1 / 3$ for which a sum of $n=2$ central Cantor sets is an interval. Cabrelli et al. [23] found, more generally, a sufficient condition for the existence of an interval in the sum of $n$ generalized Cantor sets, all of which can be constructed with a lower bound $a$ on their ratios of dissection, which is given by

$$
(n-1) \frac{a^{2}}{(1-a)^{3}}+\frac{a}{1-a} \geq 1 .
$$

\subsection{Applying Cantor set results to the Fibonacci spectra}

Before using the results quoted above to analyze the Fibonacci spectra, we should note that there exist two important differences between the energy spectra $S_{N}$ of the $N^{\text {th }}$ order approximants of the $1 d$ Fibonacci quasicrystal, and finite approximants $C_{N}$ of generalized Cantor sets. The spectrum $S_{N}$ consists of $F_{N}$ rather than $2^{N}$ energy intervals, and is not contained in the spectrum $S_{N-1}$ of the approximant of order $N-1$. One should therefore take care in defining the spectrum $S$ of the Fibonacci quasicrystal itself as the set of limit values for sequences of energies taken from consecutive spectra $S_{N}$ of finite order approximants

$$
S=\left\{E=\lim _{n \rightarrow \infty} E_{n} \mid E_{n} \in S_{n}\right\}
$$

The fact that the number of bands in $S_{N}$ is $F_{N}$ rather than $2^{N}$ implies that the spectra cannot be constructed by the iterative process described above for generalized Cantor sets, and hence that the ratios of dissection cannot be defined. However, the spectrum $S_{N}$ of a finite approximant can be padded with additional intervals which can be chosen in a manner that will not disturb the calculation, and will increase the number of intervals to $2^{N}$, as in the Cantor approximant. This allows to calculate backwards and define effective ratios of dissection. The additional intervals can be added on either, or both, ends of the spectrum. Thus, the effective ratios of dissection are not uniquely determined.

We have tried using Eq. (4) to find a sufficient condition for the higher-dimensional spectra to contain an interval. This would provide a lower bound on $T_{3}^{(n d)}$-a value of $T$ below which the condition is satisfied and the $n$-dimensional spectrum necessarily contains an interval. Unfortunately, as one studies the effective ratios of dissection defined for the $1 d$ spectrum it turns out that regardless of the way in which the approximant spectra are embedded in Cantor approximants, the ratios of dissection are not bounded away from zero, even for small values of $T$, as shown in Fig. 1(a). Hence, at this point we do not know how to use the condition of Cabrelli et al. to obtain a lower bound on $T_{3}^{(n d)}$.

Nevertheless, by studying the maximal effective ratio of dissection we can obtain an upper bound for the value of $T_{3}^{(n d)}$ above which the higher-dimensional spectra do not contain an interval. Fig. 1(b) shows the effective maximal ratio for approximants of order $N=5\left(F_{N}=8\right)$ to $N=14\left(F_{N}=610\right)$. It is evident that the maximal ratio of dissection rapidly converges as a function of the order of the 
approximant, with almost no difference between the the curves for $N=9$ and above. It is also of interest to note that the maximal ratio is independent of the way in which the approximant spectrum is embedded in a Cantor approximant. Values of $T$ for which the maximal ratio of dissection fails to satisfy Eq. (31) imply that there is no portion of the $1 d$ spectrum which can lead to the existence of an interval in the higher-dimensional spectra. The maximal ratio of dissection becomes $1 / 3$ at $T \simeq 3.15$ and $1 / 4$ at $T \simeq 4.2$. Thus, we expect to see the vanishing of intervals in the spectrum at a value of $T$ below these upper bounds for $2 d$ and $3 d$ respectively.

\section{Direct study of the $2 d$ and $3 d$ spectra}

We now turn to the direct study of the higher-dimensional spectra. This is done by explicitly calculating the spectra for approximants of finite order. Each pair or triplet of energy bands in the $1 d$ spectrum is summed to yield a single band in the $2 d$ or $3 d$ spectrum, respectively. A set of $F_{N}$ bands in the $1 d$ spectrum generates $\left(F_{N}+1\right) F_{N} / 2$ bands in the corresponding $2 d$ spectrum, and $\left(F_{N}+2\right)\left(F_{N}+1\right) F_{N} / 6$ bands in the $3 d$ spectrum, with possible overlaps that decrease as $T$ increases. Overlapping bands are merged into single energy intervals to obtain the actual structure of the higherdimensional spectra. Note that we shall use the term 'bands' to refer to the continuous energy intervals in the spectra, even though strictly speaking they may be composed of different bands with overlapping energies.

\subsection{Measuring the smallest band in the spectrum to find $T_{1}^{(n d)}$}

For $T>T_{1}^{(n d)}$ there is at least one zero-measure band in the spectrum. We therefore measure the smallest band $B_{\min }$ and ask whether it vanishes in the limit of $N \rightarrow \infty$. For $T<T_{1}^{(n d)}$ the length of the smallest band is independent of the order $N$ of the approximant. For $T>T_{1}^{(n d)}$ it can be described by a power law $B_{\min } \propto F_{N}^{-\alpha_{n}(T)}$ with some positive exponent, $\alpha_{n}(T)$. We locate $T_{1}^{(n d)}$ by finding the value of $T$ for which $\alpha_{n}$ vanishes. Fig. 2(b) clearly shows that $1.6<T_{1}^{(2 d)}<1.8$, and Fig. 2(d) indicates that $2<T_{1}^{(3 d)}<2.6$. Within these bounds, the width of the smallest band oscillates between the two different limiting behaviors.

As $T$ increases and the overlap of bands vanishes, the smallest band in the $n$-dimensional spectrum is expected to be $n$ times the smallest band of the $1 d$ spectrum. Hence for high values of $T$ the exponents $\alpha_{n}(T)$ should be independent of the dimension, because the multiplicative factor of $n$ only adds a constant term in the semi-logarithmic scale. Fig. 3 shows the extracted exponents $\alpha_{n}(T)$, indicating that they indeed coincide for all values of $T$ above $T_{1}^{(n d)}$.

\subsection{Counting the number of bands to find $T_{2}^{(n d)}$}

Next we count the number of bands $\# B$ in the spectrum and ask whether it tends to infinity or remains finite as $N$ increases. Again, we express this number as a power law of the form $\# B \propto F_{N}^{\beta_{n}(T)}$, expecting $\beta_{n}(T)$ to vanish for $T<T_{2}^{(n d)}$. For the $1 d$ Fibonacci quasicrystal $\# B_{1}=F_{N} \propto \tau^{N}$, where $\tau$ is the golden mean. In higher dimensions, as the overlap between bands decreases with increasing $T$, we expect the number of bands to tend to its maximal value, which is approximately $\left(\# B_{1}\right)^{2} / 2$ in $2 d$, and approximately $\left(\# B_{1}\right)^{3} / 6$ in $3 d$. Thus the exponents $\beta_{n}(T)$ should tend to $n \log \tau$ as $T \rightarrow \infty$. 
The dashed horizontal line in Fig. [5] indicates the expected limit value for the $2 d$ model which indeed tends to it. For the $3 d$ model the limit is only obtained at significantly higher values of $T$, indicating that the overlap of bands plays a significant role in the structure of the spectrum even at relatively high values of $T$. The continuous variation of $\beta_{2}(T)$ allows us to use smooth extrapolation and find $T_{2}^{(2 d)} \simeq 1.66$, whereas in $3 d$ we can only conclude that $2.0<T_{2}^{(3 d)}<2.6$. Combining the fact that $T_{2}^{(n d)} \geq T_{1}^{(n d)}$ with the results for the exponents $\beta_{n}(T)$ as shown in Fig. [5, we find that at least in $2 d$ and $3 d, T_{2}^{(n d)}=T_{1}^{(n d)}$, and hence that there is no intermediate regime in which the spectrum contains only a finite number of zero-measure bands.

\subsection{Measuring the largest band in the spectrum to find $T_{3}^{(n d)}$}

For $T>T_{3}^{(n d)}$ all bands in the spectrum have zero measure. We therefore look at the width of the largest band in the spectrum and ask whether it vanishes as $N \rightarrow \infty$. However, since the maximal energy in the spectrum is approximately $n(1+T)$, for small values of $T$ the overlap of bands leads to an increase in the width of largest band as a function of $T$. To avoid this we normalize the results, dividing by the maximal energy in the spectrum. Thus, for $T>T_{3}^{(n d)}$, we express the normalized largest band as a power law $B_{\max } \propto F_{n}^{-\gamma_{n}(T)}$. Figs. 6(b) and 7 clearly indicate that $T_{3}^{(2 d)} \simeq 2$, but in $3 d$ oscillatory behavior dominates a large range of values for $T$, and we cannot determine the transition without extending the analysis to higher order approximants. However, from Fig. 6(d) we can infer that the transition occurs at some value of $T$ below 5 , for which we obtained analytically a stricter upper bound of $T_{3}^{(3 d)} \leq 4.2$ as shown in Fig. 1(b).

As for $B_{\min }$, at large values of $T, B_{\max }$ is also expected to be $n$ times the largest band of the $1 d$ spectrum, and hence the exponents should be independent of dimension. The fact that this does not occur indicates, once again, that the overlap of bands is still significant for values of $T$ as large as 6 .

\subsection{Calculating the total measure of the spectrum to find $T_{4}^{(n d)}$}

To find $T_{4}^{(n d)}$ we measure the total bandwidths of the spectra as $N$ increases, normalizing by $1+T$, and looking for a power law decay of the normalized bandwidth $W \propto F_{N}^{-\delta_{n}(T)}$. Fig. 8 (c) shows a decrease in the normalized total measure of the spectrum as a function of $T$ in $2 d$, but Fig. 8 (d) shows the total measure in $3 d$ to be almost independent of $N$ for any given value of $T$. Thus, although the $3 d$ spectrum consists only of zero measure bands for values of $T$ above 5 , its total measure remains finite over the entire range of $T$ values studied. The exponents $\delta_{n}(T)$ are shown in Fig. 9, The transition to zero total bandwidth in $2 d$ occurs at $T_{4}^{(2 d)} \simeq 2$. . In $3 d$ we can only say that $T_{4}^{(3 d)}>6$.

\section{Summary and future work}

The results of Sections 2 and 3 are summarized as follows

\begin{tabular}{|l|c|c|c|c|}
\hline & $T_{1}^{(n d)}=T_{2}^{(n d)}$ & $T_{3}^{(n d)}$ & Upper bound for $T_{3}^{(n d)}$ & $T_{4}^{(n d)}$ \\
\hline $2 d$ & $\sim 1.66$ & $\sim 2$ & 3.15 & $\sim 2.6$ \\
\hline $3 d$ & $2.0-2.6$ & $\leq 5$ & 4.2 & $>6$ \\
\hline
\end{tabular}


The transitions between different regimes in the spectrum are expected to reflect on the physical properties of the Fibonacci quasicrystals, on the nature of eigenfunctions and on the dynamics of electronic wave packets. For values of $T$ above the transition $T_{3}^{(n d)}$ the higher-dimensional spectra are similar to the $1 d$ spectrum in being totally disconnected, singular continuous sets, and hence the eigenfunctions are expected to be critical, and wave packets are expected to display sub-ballistic dynamics. Note that the last transition $T_{4}^{(n d)}$ is of no consequence for this matter because the spectrum is purely singular continuous both above and below this value. For values of $T$ below the lowest transition point $T_{1}^{(n d)}=T_{2}^{(n d)}$, where the spectra are absolutely continuous we expect to find extended eigenfunctions, and wave packets are expected to display ballistic dynamics. For the intermediate range between these transitions the spectra contain both singular continuous and absolutely continuous parts, and therefore we expect to find mixed ballistic and sub-ballistic dynamics, and some of the wave functions to be extended.

We intend to complement these studies by simulating the dynamics of electronic wave functions to find whether transition between ballistic and sub-ballistic dynamics occur at the points found here. We also intend to use the degeneracy of wave functions in the $2 d$ Fibonacci quasicrystal (as hypothesized in Ref. [2]) to construct maximally extended wave functions, again, we expect to find some qualitative change in the nature of these wave functions near the transition points indicated above.

\section{Acknowledgments}

This research is supported by the Israel Science Foundation through Grant No. 684/06.

\section{References}

[1] D. Shechtman, I. Blech, D. Gratias, and J.W. Cahn, Phys. Rev. Lett. 53, 1951 (1984).

[2] R. Ilan, E. Liberty, S. Even-Dar Mandel, and R. Lifshitz, Ferroelectrics 305, 15 (2004).

[3] S. Even-Dar Mandel and R. Lifshitz, Phil. Mag. 86, 759 (2006).

[4] R. Lifshitz. J. of Alloys and Compounds 342, 186 (2002).

[5] R. Lifshitz. Z. Kristallogr. 222, 313 (2007).

[6] R. Lifshitz. Foundations of Physics 33, 1703 (2003).

[7] M. Kohmoto, L.P. Kadanoff, and C. Tang, Phys. Rev. Lett. 50, 1870 (1983).

[8] S. Ostlund, R. Pandit, D. Rand, H.S. Schellnhuber, and E.D. Siggia, Phys. Rev. Lett. 50, 1873 (1983).

[9] M. Kohmoto and J.R. Banavar, Phys. Rev. B 34, 563 (1986).

[10] M. Kohmoto, B. Sutherland, and C. Tang, Phys. Rev. B 35, 1020 (1987).

[11] T. Janssen, in The Mathematics of Long-Range Aperiodic Order, ed. R.V. Moody, (Kluwer, Dordrecht, 1997) p. 269.

[12] T. Fujiwara, in Physical Properties of Quasicrystals, ed. Z.M. Stadnik, (Springer, Berlin, 1999) ch. 6 . 
[13] J. Hafner and M. Krajcí, ibid. ch. 7.

[14] D. Damanik, in Directions in Mathematical Quasicrystals, ed. M. Baake and R.V. Moody, (AMS, Providence, 2000) p. 277.

[15] K. Ueda and H. Tsunetsugu, Phys. Rev. Lett. 58, 1272 (1987).

[16] W.A. Schwalm and M.K. Schwalm, Phys. Rev. B 37, 9524 (1988).

[17] J.X. Zhong and R. Mosseri, J. Phys: Condens. Matter 7, 8383 (1995).

[18] S. Roche and D. Mayou, Phys. Rev. Lett. 79, 2518 (1997).

[19] Yu.Kh. Vekilov, I.A. Gordeev, and E.I. Isaev, JETP 89, 995 (1999).

[20] Yu.Kh. Vekilov, E.I. Isaev, I.A. Gordeev, Mat. Sci. and Eng. 294-296, 553 (2000).

[21] J.A. Ashraff, J.-M. Luck, and R.B. Stinchcombe, Phys. Rev. B 41, 4314 (1990).

[22] G. Cantor, "De la puissance des ensembles parfait de points" (On the Power of Perfect Sets of Points), Acta Mathematica 4, 381 (1884). English translation reprinted in Classics on Fractals, ed. Gerald A. Edgar, (Addison-Wesley, 1993).

[23] C.A. Cabrelli, K.E. Hare and U.M. Molter J. Aust. Math. Soc. 73, 405 (2002).
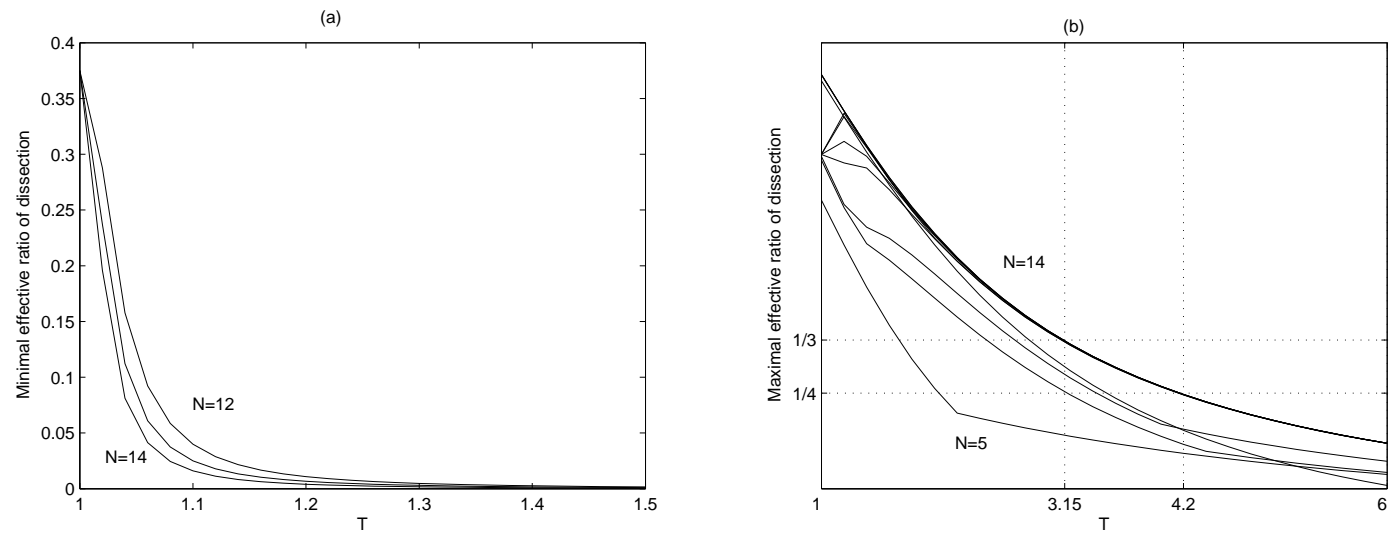

Figure 1: (a) The minimal effective ratio of dissection calculated for the $1 d$ spectra of approximants of order 12-14 for values of $T$ up to 1.5. The sharp drop near $T=1$ means that no value of $T$ satisfies the sufficient condition for obtaining an interval in the higher-dimensional spectra. (b) The maximal effective ratio of dissection calculated for the $1 d$ spectra of approximants of order 5 -14 for values of $T$ up to 6 . The horizontal dotted lines are drawn at $1 / 3$ and $1 / 4$ to indicate the upper bounds for the value of $T$ at which no intervals are to appear in the $2 d$ and $3 d$ spectra respectively. 

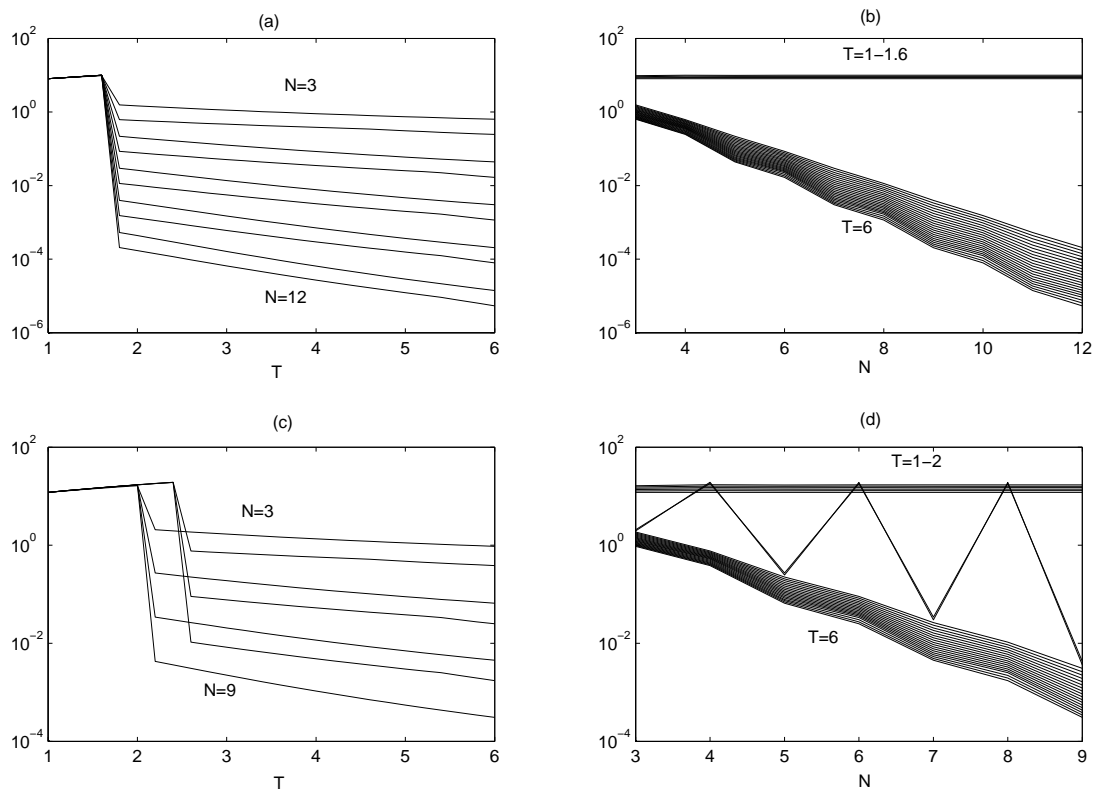

Figure 2: The length of the smallest band $B_{\min }$ in the spectrum of the $2 d$ (top) and the $3 d$ (bottom) Fibonacci quasicrystals. The length of the smallest band is plotted on the left as a function of $T$ for different approximants, and on the right as a function of $N$ for different values of $T$. The linear slopes in the semi-logarithmic plots as a function of $N$ indicate a power law behavior, $B_{\min } \propto \tau^{-N \alpha_{n}(T)}$. The exponents $\alpha_{n}(T)$ are plotted in Fig. 3 .

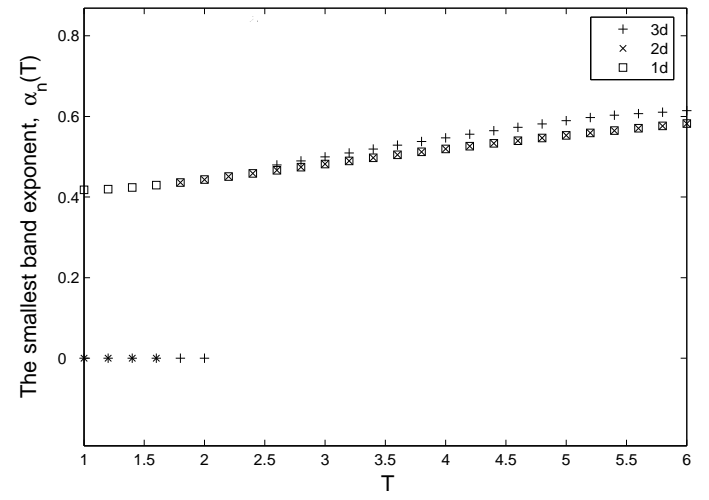

Figure 3: The exponents $\alpha_{n}(T)$ extracted from Fig. 2, The curves for $1 d, 2 d$, and $3 d$ all coincide for values of $T$ above the transition at $T_{1}^{(n d)}$. 

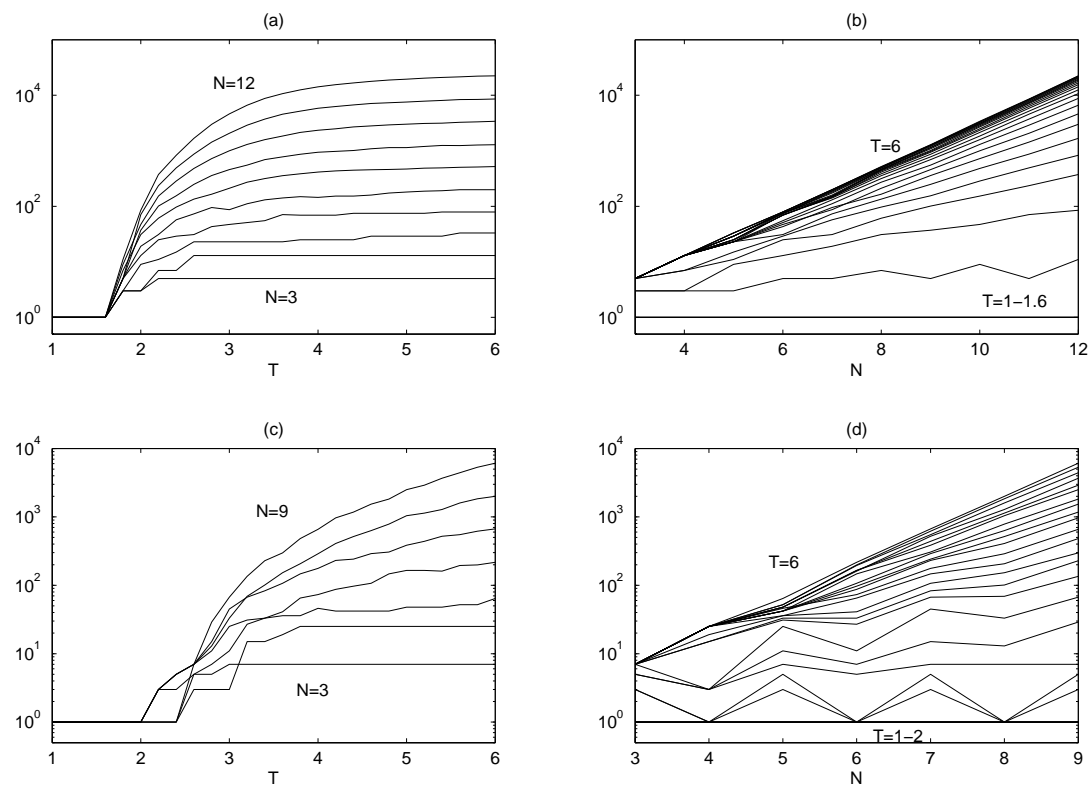

Figure 4: The number of bands \#B in the spectrum of the $2 d$ (top) and the $3 d$ (bottom) Fibonacci quasicrystals. The number of bands is plotted on the left as a function of $T$ for different approximants, and on the right as a function of $N$ for different values of $T$. The linear slopes in the semi-logarithmic plots as a function of $N$ indicate a power law behavior, $\# B \propto \tau^{N \beta_{n}(T)}$. The exponents $\beta_{n}(T)$ are plotted in Fig. 5 .



Figure 5: The exponents $\beta_{n}(T)$, extracted from Fig. 4. The horizontal dashed line indicates the expected asymptotic value of $2 \log \tau \simeq 0.418$ for the $2 d$ quasicrystal. 

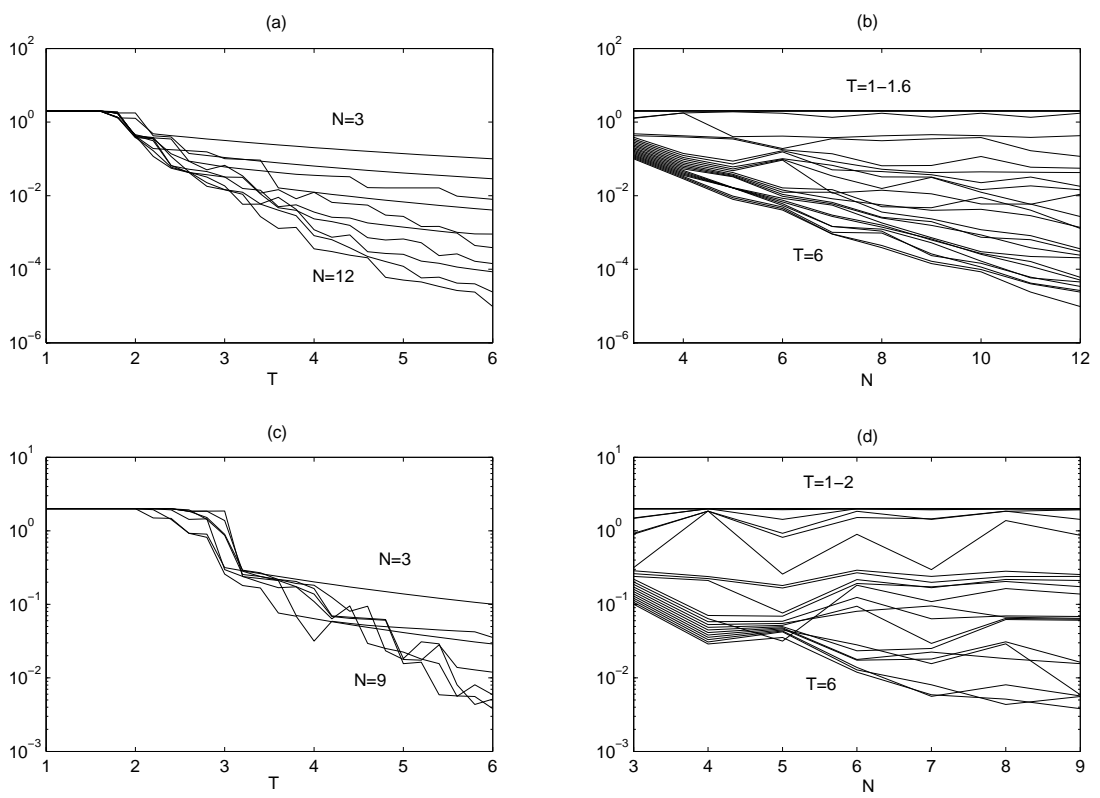

Figure 6: The normalized length $B_{\max }$ of the largest band in the spectrum of the $2 d$ (top) and the $3 d$ (bottom) Fibonacci quasicrystals. $B_{\max }$ is plotted on the left as a function of $T$ for different approximants, and on the right as a function of $N$ for different values of $T$. The linear slopes in the semi-logarithmic plots as a function of $N$ indicate a power law behavior, $B_{\max } \propto \tau^{-N \gamma_{n}(T)}$. The exponent $\gamma_{2}(T)$ is plotted in Fig. 7.

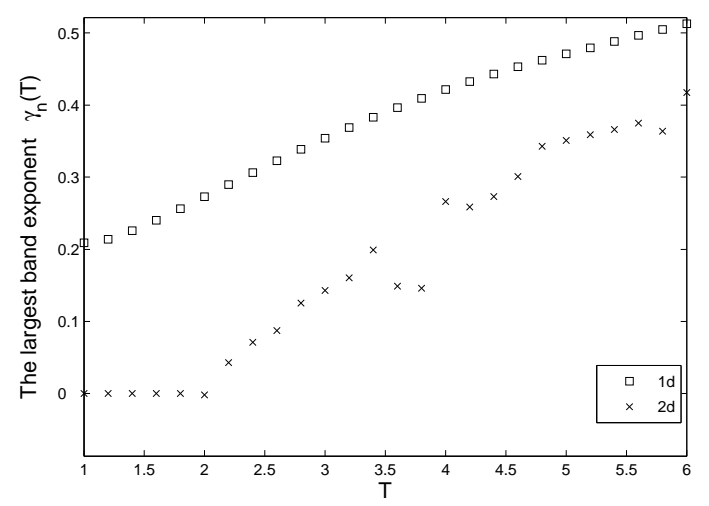

Figure 7: The exponent $\gamma_{2}(T)$ extracted from Fig. [6 and compared with $\gamma_{1}(T)$. The asymptotic behavior in which all three curves are expected to coincide is not observed for the values of $T$ shown. 



Figure 8: The normalized total bandwidth $W$ of the spectrum of the $2 d$ (top) and the $3 d$ (bottom) Fibonacci quasicrystals. $W$ is plotted on the left as a function of $T$ for different approximants, and on the right as a function of $N$ for different values of $T$. The linear slopes in the semi-logarithmic plots as a function of $N$ indicate a power law behavior, $W \propto \tau^{-N \delta_{n}(T)}$. The exponents $\delta_{n}(T)$ are plotted in Fig. 9 .

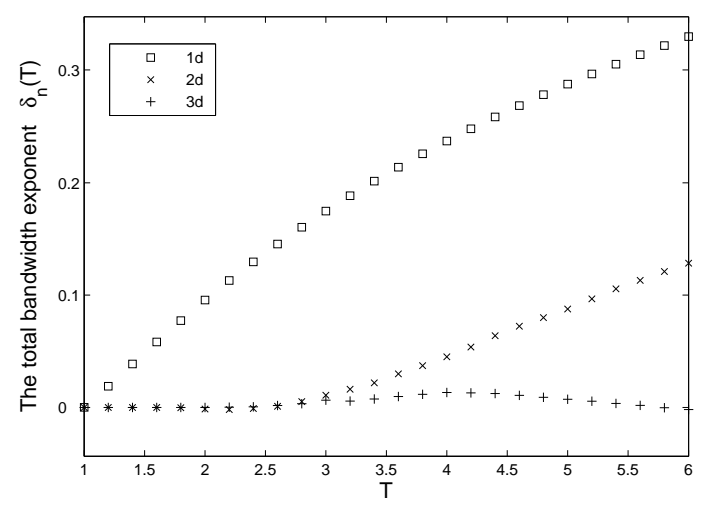

Figure 9: The exponents $\delta_{n}(T)$, extracted from Fig. 8. In $3 d$ it is evident that the transition to zero bandwidth does not occur within the studied range of $T$ values. 